\title{
Three-Dimensional Annihilation Imaging of Trapped Antiprotons
}

M. C. Fujiwara, ${ }^{1,2, *}$ M. Amoretti, ${ }^{3}$ G. Bonomi, ${ }^{4}$ A. Bouchta, ${ }^{4}$ P. D. Bowe, ${ }^{5}$ C. Carraro, ${ }^{3,6}$ C. L. Cesar, ${ }^{7}$ M. Charlton, ${ }^{5}$ M. Doser, ${ }^{4}$ V. Filippini, ${ }^{8}$ A. Fontana,${ }^{8,9}$ R. Funakoshi, ${ }^{1}$ P. Genova, ${ }^{8,9}$ J. S. Hangst, ${ }^{10}$ R. S. Hayano, ${ }^{1}$ L. V. Jørgensen, ${ }^{5}$ V. Lagomarsino, ${ }^{3,6}$ R. Landua, ${ }^{4}$ E. Lodi-Rizzini, ${ }^{8,11}$ M. Marchesotti, ${ }^{8}$ M. Macri, ${ }^{3}$ N. Madsen, ${ }^{10}$ G. Manuzio, ${ }^{3,6}$ P. Montagna, ${ }^{8,9}$ P. Riedler, ${ }^{4}$ A. Rotondi, ${ }^{8,9}$ G. Rouleau, ${ }^{4,5}$ G. Testera, ${ }^{3}$ A. Variola, ${ }^{3}$ D. P. van der Werf, ${ }^{5}$ and Y. Yamazaki ${ }^{2}$

\section{(ATHENA Collaboration)}

\author{
${ }^{1}$ Department of Physics, University of Tokyo, Tokyo 113-0033, Japan \\ ${ }^{2}$ Atomic Physics Laboratory, RIKEN, Saitama 351-0198, Japan \\ ${ }^{3}$ Istituto Nazionale di Fisica Nucleare, Sezione di Genova, I-16146 Genova, Italy \\ ${ }^{4}$ EP Division, CERN, Geneva, Switzerland \\ ${ }^{5}$ Department of Physics, University of Wales Swansea, Swansea SA2 8PP, United Kingdom \\ ${ }^{6}$ Dipartimento di Fisica di Genova, I-16146 Genova, Italy \\ ${ }^{7}$ Instituto de Fisica, Universidade Federal do Rio de Janeiro, Rio de Janeiro 21945-970, Brazil \\ ${ }^{8}$ Istituto Nazionale di Fisica Nucleare Sezione di Pavia, I-27100 Pavia, Italy \\ ${ }^{9}$ Dipartimento di Fisica Nucleare e Teorica, Università di Pavia, I-27100 Pavia, Italy \\ ${ }^{10}$ Department of Physics and Astronomy, University of Aarhus, DK-8000 Aarhus C, Denmark \\ ${ }^{11}$ Dipartimento di Chimica e Fisica per l'Ingegneria e per i Materiali, I-25123 Brescia, Italy
}

(Received 31 July 2003; published 13 February 2004)

\begin{abstract}
We demonstrate three-dimensional imaging of antiprotons in a Penning trap, by reconstructing annihilation vertices from the trajectories of the charged annihilation products. The unique capability of antiparticle imaging has allowed, for the first time, the observation of the spatial distribution of the particle loss in a Penning trap. The radial loss of antiprotons on the trap wall is localized to small spots, strongly breaking the azimuthal symmetry expected for an ideal trap. Our observations have important implications for detection of antihydrogen annihilations.
\end{abstract}

DOI: 10.1103/PhysRevLett.92.065005

PACS numbers: 52.27.Jt, 36.10.-k, 39.10.+j

Charged elementary particles and ions can be contained in an electromagnetic trap, known as a Penning trap, which confines the radial motion by a magnetic field and the axial motion by an electric field [1]. Penning traps have been used in many areas of both pure and applied research, from precision spectroscopy to quantum information, as well as for antimatter confinement $[2,3]$.

While various diagnostic techniques exist for trapped particle and plasma studies, imaging can give direct information about the particle cloud properties. A common technique of dumping the particles on a phosphor screen or collimated Faraday cup [3] gives the radial cloud profile (perpendicular to the magnetic field axis), but the axial information is integrated out. For studies of trapped atomic ions, laser fluorescence techniques can be used for imaging [4], if convenient transition lines exist, yet this method is not applicable to elementary (anti)particles. In this Letter, we demonstrate three-dimensional imaging of trapped antiprotons by reconstructing their annihilation vertices using the ATHENA apparatus [5-9]. Depending on the residual gas densities and particle dynamics, the annihilation can take place either on the gas or on the trap wall. We focus our attention on radial loss processes, exploiting the distinctive capability of imaging anti- matter particles. In a sufficiently high vacuum, where annihilation on the residual gas is negligible, antiparticle annihilation imaging has unique sensitivity to losses at the trap walls. No other techniques have yet allowed such direct observations of charged particle losses in traps.

Beginning with pioneering works in the 1980s [10], radial particle transport across the magnetic field lines in a trapped plasma has been the subject of considerable research [10,11], since it sets a practical limit on the plasma confinement time. O'Neil's confinement theorem [12] states that, for an axially symmetric system, due to the conservation of canonical angular momentum, the mean-square radius of the ensemble of charged particles is approximately conserved. It is now well established that radial transport in the low pressure regime is driven by small trap imperfections and field misalignments that break the axial symmetry of the system [11]. However, little attention has been paid to the particle dynamics in the proximity of the wall. With the first direct observation of particle losses at the wall, we probe this previously unexplored final step of the radial transport, and show that antiproton radial loss occurs in a manner that is highly nonuniform both axially and radially. In addition to their importance for antihydrogen production and 


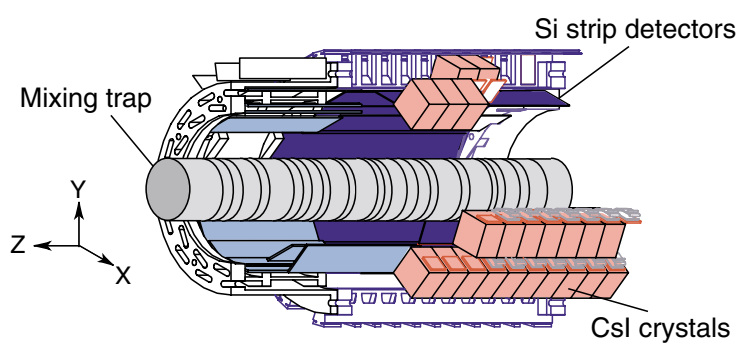

FIG. 1 (color online). A schematic view of the ATHENA vertex detector and the mixing trap. The trap consists of a series of hollow cylindrical electrodes (inner radius $1.25 \mathrm{~cm}$ ), and is held at a temperature between 15 and $40 \mathrm{~K}$. The detector is kept at $140 \mathrm{~K}$ and is housed in a separate vacuum. Both are in a $3 \mathrm{~T}$ magnetic field.

detection, our observations may apply to charged particle loss in Penning traps in general.

The ATHENA apparatus, which recently produced the first cold antihydrogen atoms [5], is described elsewhere [5-9,13]; hence, only a brief description is given here. The Antiproton Decelerator facility located at CERN provides typically $2 \times 10^{7}$ antiprotons having kinetic energy of $5.3 \mathrm{MeV}$ every $100 \mathrm{~s}$. They are slowed in a degrader foil and trapped dynamically using a pulsed electric field. Preloaded cold electrons in a $3 \mathrm{~T}$ magnetic field cool the antiprotons by Coulomb collisions from $\mathrm{keV}$ to $\mathrm{meV}$ energies $[14,15]$. For the measurements reported here, $10^{3}$ to $10^{4}$ cold antiprotons, together with $10^{7}$ to $10^{8}$ electrons, are held in the central trap region (the so-called mixing trap, Fig. 1) where they may annihilate. A measurement cycle of $100 \mathrm{~s}$ duration is repeated, and annihilation images are obtained by summing over many cycles.

Annihilations of antiprotons, either on the trap wall or on residual gas atoms, produce about three charged pions on average, and these are detected by two layers of double-sided Si microstrip detectors [7] which subtend $80 \%$ of the solid angle (Fig. 1). Signals from a total of 8192 detector channels are read out into flash analog-todigital converters, which in turn are read into a personal computer and recorded on disk. The detector readout (rate $<40 \mathrm{~Hz}$ ) is triggered by a coincidence of two or more charged particle signals (hits) in the outer Si layer. Hits in the two Si layers are fitted with a straight line to reconstruct a charged track and, from the intersection of two or more tracks, the three-dimensional position of the annihilation vertex is obtained. The unmeasured curvature of the charged particles in the $3 \mathrm{~T}$ magnetic field, together with multiple scattering in the structure of the apparatus, limits our vertex position resolution to $\sim 4 \mathrm{~mm}(1 \sigma)$.

Figure 2(a) shows an image of antiproton annihilations in a harmonic potential well of depth $30 \mathrm{~V}$ and length $5 \mathrm{~cm}$. With a relatively high gas pressure of the order of $10^{-11}$ mbar (estimated from the antiproton storage lifetime of a few hundred seconds [16]), annihilation on residual gas atoms (or ions) dominates. The image thus reflects the profile of the trapped antiprotons at the time of
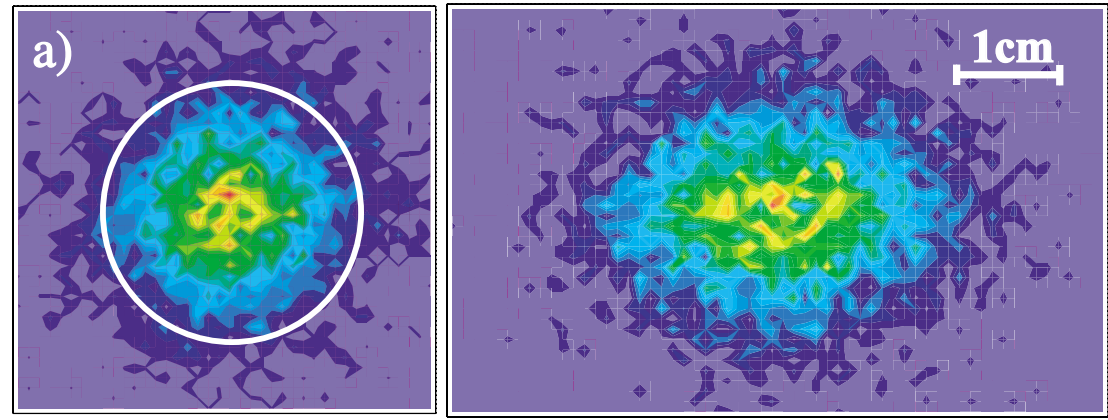

0

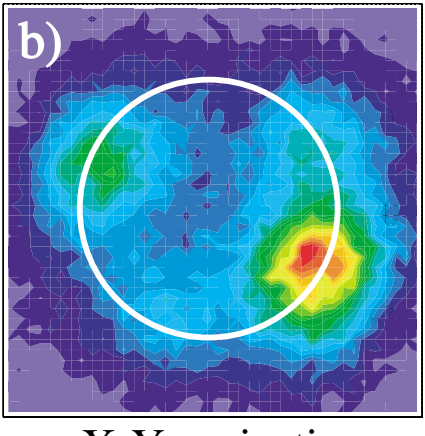

$\mathrm{X}-\mathrm{Y}$ projection

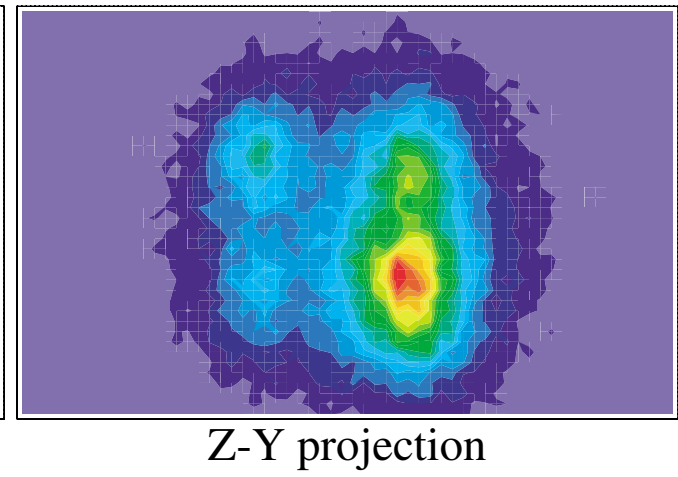

FIG. 2 (color). Annihilation image of antiprotons trapped in a harmonic trap for (a) high pressure and (b) low pressure. The harmonic potential is formed with five central and two end cap electrodes. The estimated position of the trap wall is depicted with a white line. The same contour scheme (linear scale) is used throughout this Letter. 

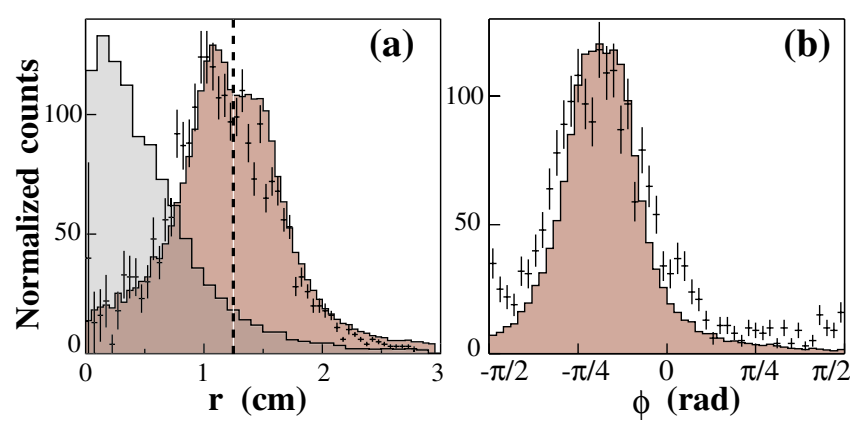

FIG. 3 (color). (a) Comparison of radial ( $r$ ) annihilation distributions $(d N / r d r)$ for the data from the low pressure measurement (error bars) and the MC simulations assuming annihilations on the trap wall (brown histogram). The inner radius of the trap electrodes is indicated with a dashed line. Also shown are the data for the high pressure measurement (grey histogram). (b) The azimuthal $(\phi)$ angular distribution of the annihilation (error bars) and its comparison with the MC assuming point source annihilation.

their annihilation, an axially symmetric distribution as expected.

Striking features in the annihilation pattern are observed in Fig. 2(b), where the residual gas density is reduced to less than $10^{-13}$ mbar with other conditions kept similar. In this case, the radial loss on the wall dominates over annihilation on the gas. The observed annihilation distribution is strongly anisotropic, and is localized to a few "hot spots." Indeed, as we shall show, the localization of antiproton loss at the trap wall is a general feature observed in the high vacuum regime.

Detailed Monte Carlo (MC) simulation studies were performed in order to quantitatively understand the images obtained. The MC code, based on the GEANT package [17], simulates the interaction of the annihilation products with the detector and the surrounding materials. It utilizes the branching ratios and the decay phase space for antiproton annihilations on protons, and simulates full electromagnetic and hadronic cascades. The detector geometry and its module-by-module efficiencies are taken into account. Generated events are passed through the same analysis program as the annihilation vertex reconstruction. Figure 3(a) compares the radial distribution for the high vacuum case [Fig. 2(b)] and that from the MC assuming a point source of annihilation on the trap wall. The good agreement establishes that most of the annihilations are indeed occurring at the wall. Also shown is the radial distribution for the high pressure case [Fig. 2(a)],

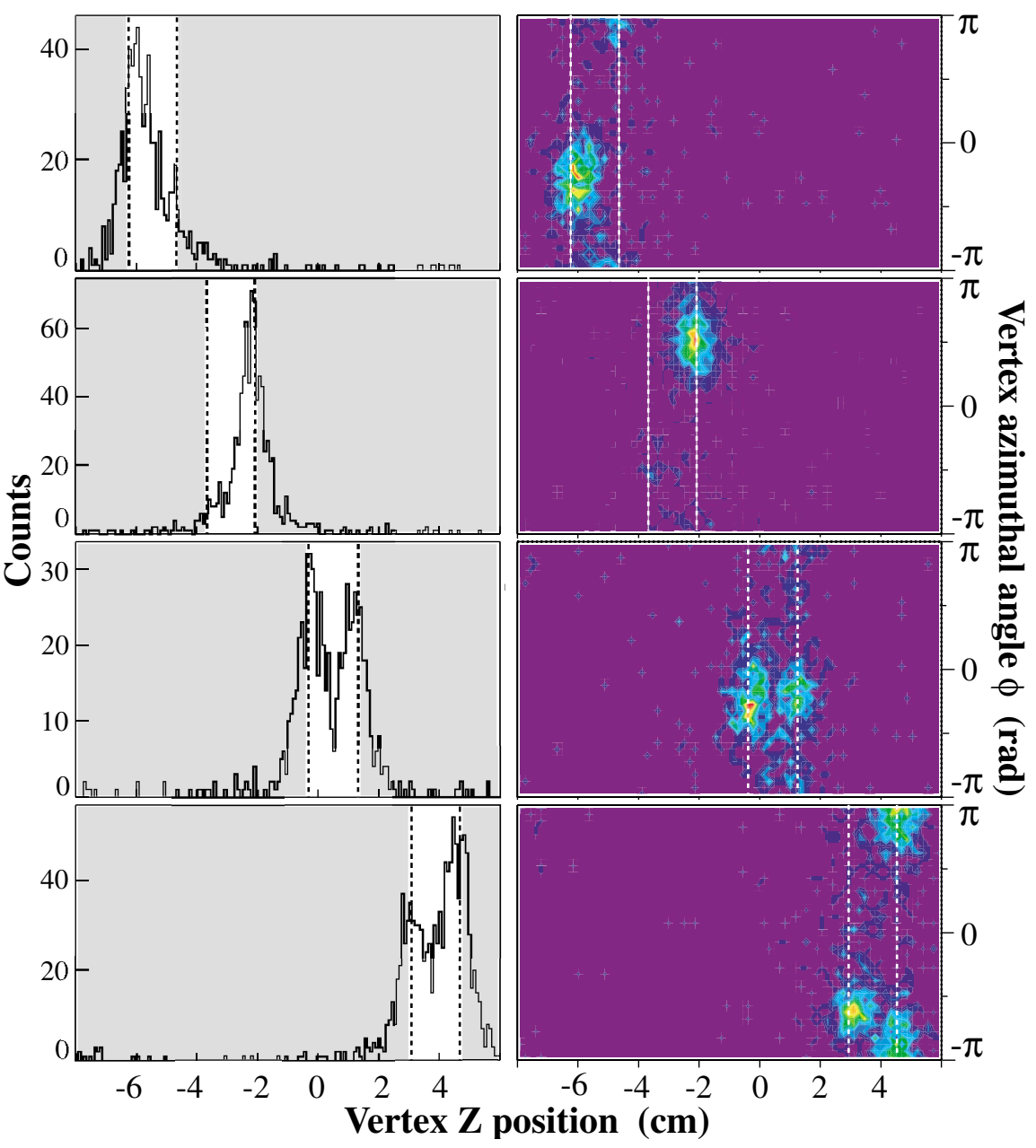

FIG. 4 (color). The projection of the annihilation distribution on the $z$ axis (left column) and on the $z-\phi$ plane (right column) for four different confinement setups. The trap well positions are indicated by the unshaded regions, and the dimensions of the electrodes are depicted with dashed lines. 
which is clearly distinguishable from that representing annihilation on the wall.

In order to study the degree of annihilation localization, we focus on one of the observed hot spots (by making spatial cuts in our vertex distribution, both in the $z$ coordinate and in the $x-y$ plane), and compare its azimuthal distribution with that of an MC-generated point source. From the reasonable agreement shown by the comparison given in Fig. 3(b), together with further MC simulations incorporating an extended source [18], we can exclude a hot spot size larger than $8 \mathrm{~mm}$.

The localization of the antiproton loss at the wall is observed in the high vacuum regime, irrespective of other trap conditions. We illustrate this in the following two series of examples. Figure 4 shows antiprotons trapped in a well formed by a single electrode, while Fig. 5 pertains to antiprotons trapped in wells having various numbers of electrodes. A fixed potential, with respect to the rest of the grounded electrodes, of either $+50 \mathrm{~V}$ to $+140 \mathrm{~V}$ was applied to the well electrode(s) (unshaded region Figs. 4 and 5). The exact value of this potential does not affect the characteristics of the distributions. In both series, the annihilation distributions are strongly correlated with the trap well positions, as expected. The observed annihilations, however, are highly nonuniform both axially and azimuthally, as before. In the $z$ projection, annihilations are seen to cluster mainly at the edges of the electrodes.
The number of hot spots grows with the number of the electrodes used in the well. A four-sector, azimuthally segmented electrode in some cases produces stronger annihilation spots (e.g., top panel, Fig. 5) [19].

Various other measurements performed all showed localized annihilations on the wall, regardless of the details of the antiproton catching method, the electron (re)loading procedure, and the value of the well depth. The locations of the hot spots are reproducible for the same trap conditions, although we have observed changes in their relative amplitudes on a time scale of several weeks. Two separate trap systems both showed hot spots.

It is worthwhile quoting our estimates of trap asymmetries and antiproton dynamical time scales. The trap electrodes, made of aluminum, have a fabrication out-ofround (ovality) tolerance of $\leq 20 \mu \mathrm{m}$, and an alignment tolerance in assembly of $\leq 50 \mu \mathrm{m}$. The electrodes have a tooling surface roughness $\leq 1 \mu \mathrm{m}$ (N6 grade), and are covered with $2 \mu \mathrm{m}$ of electrodeposited gold. The effect of surface charge was investigated by intentionally applying asymmetric potentials on one of the azimuthally sectored electrodes and measuring the reduction in the electron storage time. Effects greater than $100 \mathrm{mV}$ can be excluded. In our central trap region, the magnetic field homogeneity is better than $5 \times 10^{-4}$, and the mechanical misalignment of the trap axis (with respect to the magnet bore) is less than $1 \mathrm{mrad}$. In the reported measurements,

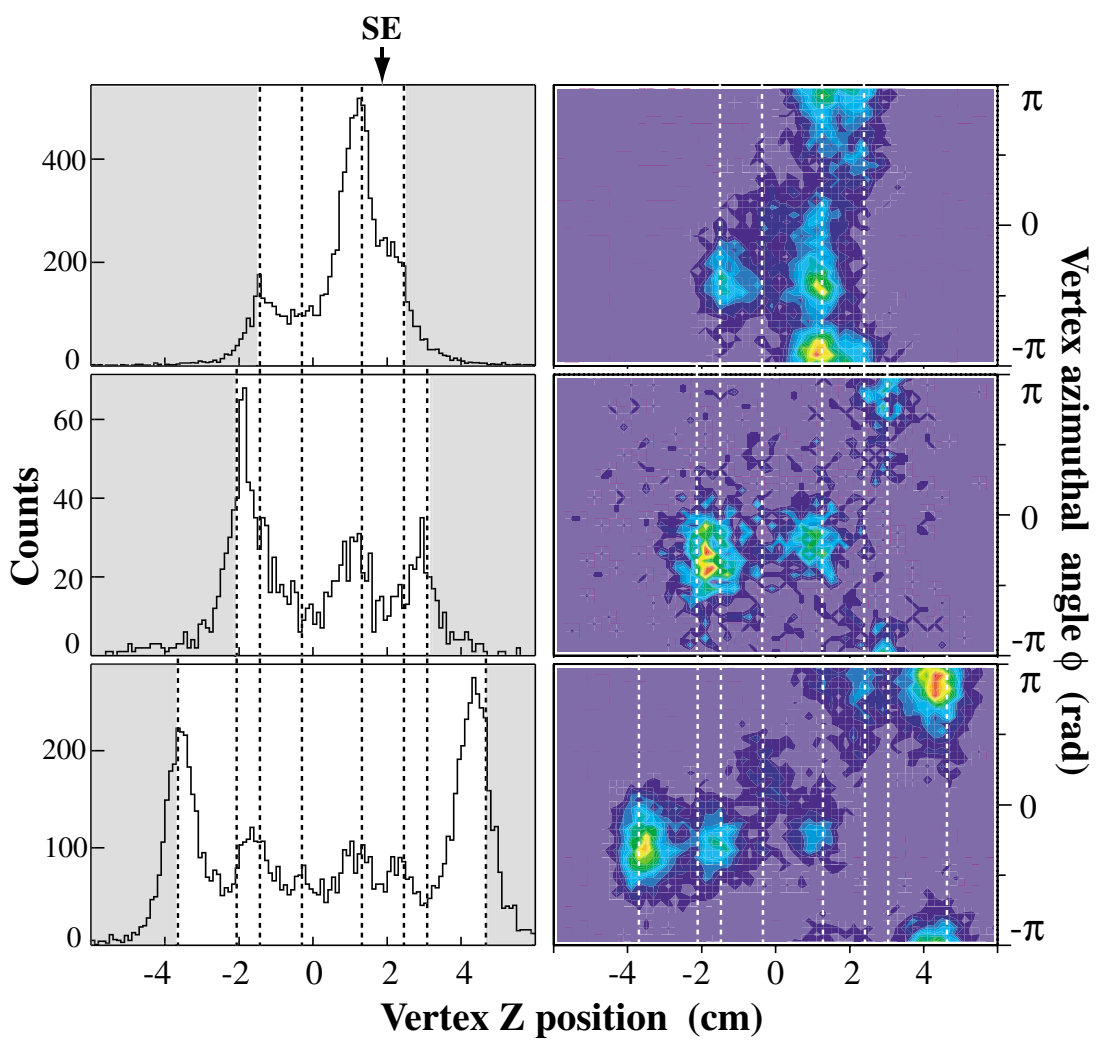

FIG. 5 (color). Similar to Fig. 4, but with varying numbers of electrodes used in the trap well, shown as unshaded regions. "SE" indicates the fourfold azimuthally sectored electrode. 
assuming antiprotons are in complete overlap and in thermal equilibrium with the electron plasma at 15$40 \mathrm{~K}$, their axial bounce frequency $f_{b} / 2 L$ is in the range $2 \mathrm{kHz} \lesssim f_{b} \lesssim 20 \mathrm{kHz}$ with $1.5 \mathrm{~cm} \leq L \leq 8 \mathrm{~cm}$, and the $E \times B$ drift frequency [20] $2 \mathrm{kHz} \lesssim f_{E \times B} \lesssim 120 \mathrm{kHz}$. Note that the antiproton cyclotron frequency is $46 \mathrm{MHz}$ and the radius $1.7-2.8 \mu \mathrm{m}$ in a $3 \mathrm{~T}$ magnetic field, and the single particle axial and magnetron frequencies for our harmonic trap are 490 and $2.6 \mathrm{kHz}$, respectively.

With most of the electrons in the trap removed [21], antiproton radial transport is greatly altered and generally suppressed, making it harder to obtain annihilation images on the wall, especially for a short harmonic potential, in which the storage time exceeds $10 \mathrm{~h}$ [15]. Loss images could be obtained in a longer trap (length $\sim 10 \mathrm{~cm}$ ) with the electrons removed, and they do indeed show hot spots. The existence of hot spots even with few electrons (or with other trap conditions mentioned above) suggests that the localization mechanism is less dependent on the transport process in the plasma bulk than on the particle dynamics in the proximity of the wall surface. One relevant effect may be small off-axis displacements of two adjacent electrodes, which would exert an $E \times B$ force on antiprotons towards the wall due to the nonradial electric field at the electrode junctions. Clearly, more studies are needed in order to pin down the mechanism for loss localization reported in this Letter.

The measurements reported here have significant practical implications for our detection of antihydrogen annihilation. In Ref. [5], we reported production of cold antihydrogen by detecting the simultaneous annihilations of the positron and the antiproton on the trap wall. While the antiproton annihilation vertices can be reconstructed with an efficiency of about $50 \%$ of all of the antihydrogen events, the detection of back-to-back $511 \mathrm{keV} \gamma$ from positron annihilations is much more difficult, due to the low efficiency of the $\gamma$ detection, the lower signal-tonoise, and the presence of physical backgrounds. Our observation that the (charged) antiproton losses on the wall are localized to hot spots, while the neutral antihydrogen annihilates in a radially symmetrical manner (see Fig. 3 of Ref. [5]), can provide a new and effective signature of antihydrogen annihilation, based on the charged vertices alone (without relying on the $511 \mathrm{keV}$ $\gamma$ detection). If this applies to other trap systems, it could substantially simplify the detection system necessary in future antihydrogen experiments.

We thank CERN's AD crew staff for providing antiproton beams, J. Rochet and the University of Zurich group for detector development, and H. Higaki and A. Mohri for valuable discussions. This work was supported in part by MEXT and RIKEN (Japan), CNPq (Brazil), SNF (Denmark), INFN (Italy), SNF (Switzerland), and the EPSRC (U.K.).

*Author to whom correspondence should be addressed. Electronic address: Makoto.Fujiwara@cern.ch

[1] H. Dehmelt, Rev. Mod. Phys. 62, 525 (1990); L. S. Brown and G. Gabrielse, ibid. 58, 233 (1986).

[2] P. K. Ghosh, Ion Traps (Oxford University Press, Oxford, 1995).

[3] D. H. Dubin and T M. O’Neil, Rev. Mod. Phys. 71, 87 (1999); Non-Neutral Plasma Physics, edited by F. Anderegg (AIP, New York, 2002), Vol. 4.

[4] D. J. Larson et al., Phys. Rev. Lett. 57, 70 (1986); L. R. Brewer et al., Phys. Rev. A 38, 859 (1988); T. B. Mitchell et al., Opt. Express 2, 314 (1998).

[5] M. Amoretti et al., Nature (London) 419, 456 (2002).

[6] M. Amoretti et al., Nucl. Instrum. Methods Phys. Res., Sect. A 518, 679 (2004).

[7] C. Regenfus, Nucl. Instrum. Methods Phys. Res., Sect. A 501, 65 (2003).

[8] M. C. Fujiwara et al., Nucl. Instrum. Methods Phys. Res., Sect. B 214, 11 (2004); hep-ex/0401039 (to be published).

[9] M. Amoretti et al., Phys. Rev. Lett. 91, 055001 (2003); Phys. Plasmas 10, 3056 (2003).

[10] J. H. Malmberg and C. F. Driscoll, Phys. Rev. Lett. 44, 654 (1980); C. F. Driscoll and J. H. Malmberg, ibid. 50, 167 (1983); C. F. Driscoll et al., Phys. Fluids 29, 2015 (1986).

[11] J. Notte and J. Fajans, Phys. Plasmas 1, 1123 (1994); D. L. Eggleston and T. M. O’Neil, ibid. 6, 2699 (1999); E. H. Chao et al., ibid. 7, 831 (2000); J. M. Kriesel and C. F. Driscoll, Phys. Rev. Lett. 85, 2510 (2000); A. A. Kabantsev et al., ibid. 87, 225002 (2001); E. Sarid et al., ibid. 89, 105002 (2002); A. A. Kabantsev and C. F. Driscoll, ibid. 89, 245001 (2002); A. A. Kabantsev et al., Phys. Plasmas 10, 1628 (2003).

[12] T. M. O’Neil, Phys. Fluids 23, 2216 (1980).

[13] M. Amoretti et al., Phys. Lett. B 578, 23 (2004).

[14] G. Gabrielse et al., Phys. Rev. Lett. 57, 2504 (1986); 63, 1360 (1989).

[15] M. C. Fujiwara et al., Hyperfine Interact. 138, 153 (2001).

[16] X. Fei, Ph.D. thesis, Harvard University, 1990. Note that this assumes theoretical low energy annihilation cross sections which are untested.

[17] CERN Application Software Group, GEANT, CERN Program Library W5013, Geneva (1993).

[18] M. C. Fujiwara et al., Non-Neutral Plasma Physics, edited by M. Schauer et al. (AIP, New York, 2003), Vol. 5, p. 131.

[19] We note that early observations on particle transport reported reduction in the plasma lifetime for longer traps and for traps including sectored electrodes [10].

[20] K. S. Fine and C. F. Driscoll, Phys. Plasmas 5, 601 (1998).

[21] The upper limit of the number of remaining electrons after removal is $10^{5}$, as dictated by the sensitivity of the detection system. 